03,05

\title{
Смена знака магнитоемкости в парамагнитной области в катион-замещенном селениде марганца
}

\author{
(C) С.С. Аплеснин ${ }^{1.2}$, М.Н. Ситников ${ }^{1}$, А.М. Живулько \\ ${ }^{1}$ Сибирский государственный университет науки и технологий им. акад. М.Ф. Решетнева, \\ Красноярск, Россия \\ ${ }^{2}$ Институт физики им. Л.В. Киренского, ФИЦ КНЦ СО РАН, \\ Красноярск, Россия \\ ${ }^{3}$ НПЦ НАН Беларуси по материаловедению, \\ Минск, Беларусь \\ E-mail: apl@iph.krasn.ru
}

(Поступила в Редакцию 27 сентября 2017 г.

В окончательной редакции 18 октября 2017 г.)

\begin{abstract}
В твердом растворе $\mathrm{Gd}_{x} \mathrm{Mn}_{1-x} \mathrm{Se}(x \leq 0.2)$ проведены измерения емкости и тангенса угла диэлектрических потерь в интервале частот $1-300 \mathrm{kHz}$ без магнитного поля и в магнитном поле $8 \mathrm{kOe} \mathrm{в} \mathrm{интервале}$ температур $100-450 \mathrm{~K}$, магнитного момента в поле $8.6 \mathrm{kOe}$. Обнаружен магнитоемкостный эффект и смена знака магнитоемкости в области комнатных температур в парамагнитной области. Установлена корреляция изменения диэлектрической проницаемости и магнитной восприимчивости по температуре. Магнитоемкость описывается в моделях с орбитальным упорядочением электронов и Максвелла-Вагнера.
\end{abstract}

Работа выполнена при финансовой поддержке РФФИ N17-32-50080, гранта № 16-52-00045 Бел_а и государственного задания № 3.5743.2017/6.7.

DOI: 10.21883/FTT.2018.04.45673.286

\section{1. Введение}

Материалы, в которых проявляется взаимосвязь магнитных и электрических свойств [1,2], магнитоэлектрики и мультиферроики [3,4], представляют интерес как с фундаментальной, так и с прикладной точек зрения. Особое внимание привлекают материалы, обнаруживающие магнитоэлектрические свойства в области комнатных и более высоких температур в связи с практическим применением в микроэлектронике для записи и хранения информации. К таким изученным материалам относится феррит висмута $\mathrm{BiFeO}_{3}[5,6]$. Эффект гигантской магнитоемкости наблюдался в $\mathrm{LuFe}_{2} \mathrm{O}_{4}$ [7] при комнатной температуре и объясняется флуктуацией заряда с разной величиной спина в ионах $\mathrm{Fe}^{2+}$ и $\mathrm{Fe}^{3+}$ в результате снятия вырождения между двумя типами зарядового порядка внешним магнитным полем. Зависимость орбитальных магнитных моментов от полярных искажений, индуцированных под действием электрического поля, дает „ионно-орбитальный“ вклад в магнитоэлектрический отклик [8].

Системы с орбитальным упорядочением характеризуются анизотропным распределением электронной плотности в решетке с отличающимися параметрами интегралов перескока $[9,10]$ и соответственно анизотропией подвижности носителей тока. Поэтому миграционная электронная поляризация также является анизотропной. При наличии орбитального магнитного момента, например у электронов в $t_{2 g}$-состояниях, анизотропией диэлектрической проницаемости можно управлять магнитным полем.
В электрически неоднородных системах эффект Максвелла-Вагнера [11] и контактные эффекты могут привести к гигантским значениям диэлектрической проницаемости и диэлектрической релаксации в отсутствие дипольной релаксации [12]. Эффект Максвелла-Вагнера также может индуцировать магнитоемкость в отсутствие взаимодействия между магнитной и электрической подсистемами, при условии существования магнитосопротивления в материале [13]. Такие эффекты ясно демонстрируют, что наличие магнитоемкости не достаточно для отнесения этих соединений к мультиферроикам. С другой стороны, магнитоемкость без магнитоэлектрической связи может быть более практичной для технологических применений, так как не требуется существования дальнего магнитного порядка.

Электрически неоднородную систему с орбитальным вырождением можно получить путем замещения двухвалентных ионов марганца трехвалентными ионами гадолиния. Так, исходные соединения селенид марганца и селенид гадолиния относятся соответственно к полупроводникам и вырожденным полуметаллам, электрическое сопротивление которых отличается в $10^{6}-10^{9}$ раз [14]. Селенид гадолиния и селенид марганца имеют антиферромагнитную структуру 2-го типа упорядочения с температурой Нееля $T_{N}=63 \mathrm{~K}[15]$ и $T_{N}=137 \mathrm{~K}[16]$ соответственно. Спин-орбитальное и ян-теллеровское взаимодействия снимают вырождение $t_{2 g}$-электронных состояний и индуцируют расщепление спектра электронных возбуждений по спину. В результате диэлектри- 

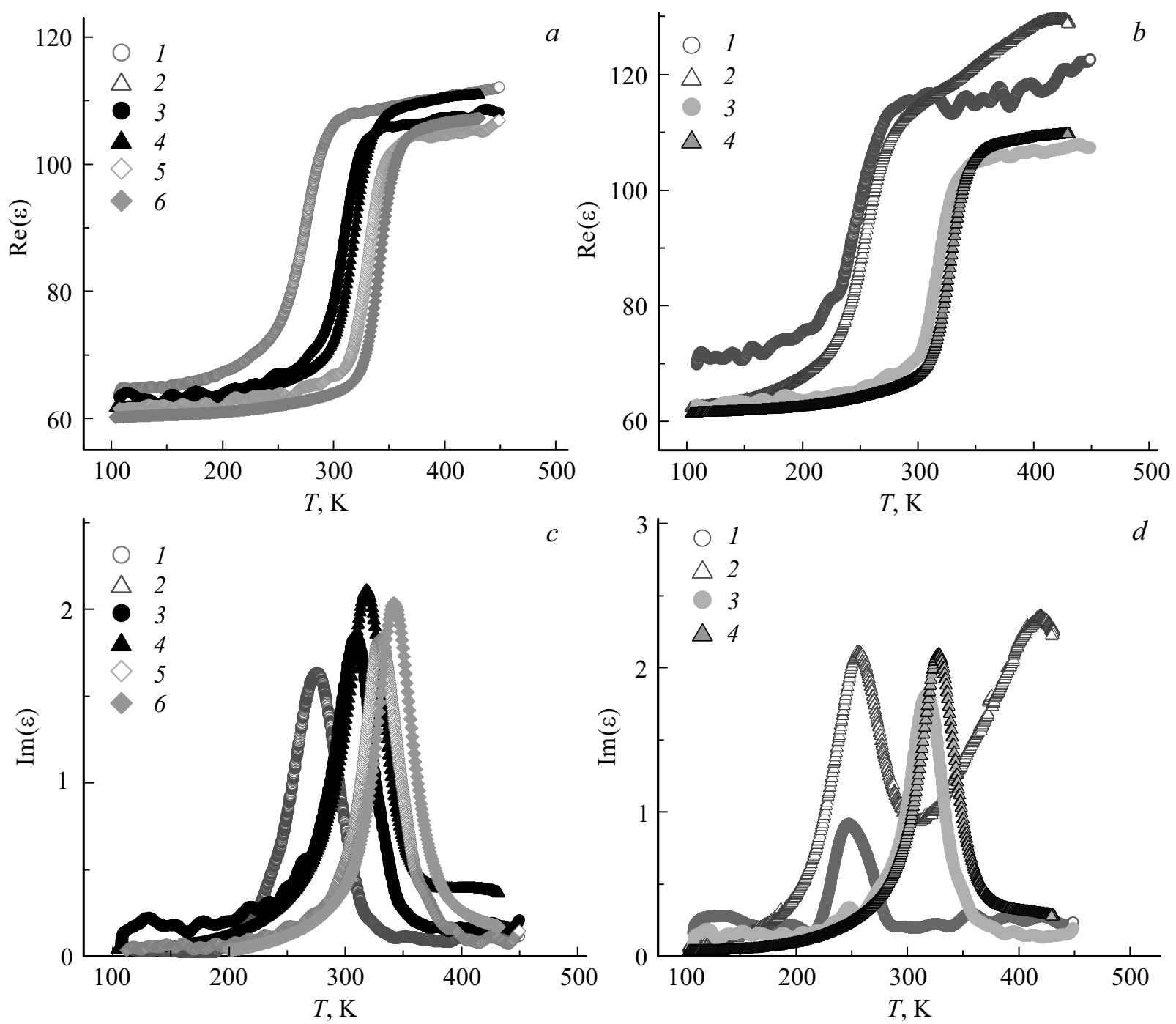

Рис. 1. Действителььная $\operatorname{Re}(\varepsilon)(a, b)$ и мнимая $\operatorname{Im}(\varepsilon)(c, d)$ компоненты диэлектрической проницаемости образца $\mathrm{Gd}_{0.05} \mathrm{Mn}_{0.95} \mathrm{Se}_{\mathrm{B}}$ магнитном поле $H=0(1,3.5), H=8 \mathrm{kOe}(2,4,6)$ на частотах $\omega=5,50,300 \mathrm{kHz}(a, c), \omega=1,100 \mathrm{kHz}(b, d)$ от температуры.

ческими свойствами можно управлять электрическим и магнитным полями.

Цель данной работы - установить вклад в магнитоемкость от орбитального упорядочения электронов и эффекта Максвелла-Вагнера в спин-неупорядоченной области в твердых растворах $\mathrm{Gd}_{x} \mathrm{Mn}_{1-x} \mathrm{Se}$.

\section{2. Экспериментальные результаты и обсуждение}

Твердые растворы $\mathrm{Gd}_{x} \mathrm{Mn}_{1-x} \mathrm{Se}$ получены методом твердофазной реакции, описанной в работе [17], из порошков исходных соединений в вакуумированных кварцевых ампулах в однозонной печи сопротивления. Определение фазового состава и кристаллической структуры образцов $\mathrm{Gd}_{X} \mathrm{Mn}_{1-X} \mathrm{Se}$ проведено при $300 \mathrm{~K}$ на рентгеновской установке ДРОН-3 с использованием $\mathrm{Cu} K_{\alpha}$-излучения. Данные рентгеноструктурного анализа показали, что синтезированные соединения обладают гранецентрированной кубической (ГЦК) структурой типа $\mathrm{NaCl}$, типичной для моноселенида марганца. C увеличением степени катионного замещения $(X)$ параметр элементарной ячейки $a$ увеличивается от $a=0.5440 \mathrm{~nm}$ для $\mathrm{MnSe}$ до $a=0.5520 \mathrm{~nm}$ для $\mathrm{Gd}_{0.2} \mathrm{Mn}_{0.8} \mathrm{Se}$ [17].

Емкость и тангенс угла диэлектрических потерь $(\operatorname{tg} \delta)$ измерены на анализаторе компонентов АМ-3028 в интервале температур 90-450 K без магнитного поля и в магнитном поле $H=8 \mathrm{kOe}$. Магнитное поле прикладывалось параллельно пластинам плоского конденсатора. Магнитоемкостный эффект $\delta \varepsilon_{H}=(\operatorname{Re}(\varepsilon(H, T))-\operatorname{Re}(\varepsilon(0, T))) / \operatorname{Re}(\varepsilon(0, T))$ определяется в результате исследования комплексной диэлектрической проницаемости. Спектральные и температурные зависимости диэлектрических констант можно использовать для обнаружения дипольного электрического момента и определения его характеристик, даже когда речь идет о локальном дипольном моменте в малых 
кластерах без наличия дальнего порядка. Диэлектрические свойства отражают также информацию о зарядовом транспорте и процессах зарядового упорядочения. Отклик диэлектрических свойств на воздействие магнитного поля даст возможность определить основные механизмы, определяющие взаимосвязь диэлектрических и магнитных свойств.

На рис. 1 изображены температурные зависимости действительной $\operatorname{Re}(\varepsilon)$ и мнимой $\operatorname{Im}(\varepsilon)=\operatorname{tg} \delta \operatorname{Re}(\varepsilon)$ частей диэлектрической проницаемости образца $\mathrm{Gd}_{0.05} \mathrm{Mn}_{0.95} \mathrm{Se}$. В интервале температур 220-330 K реальная часть диэлектрической проницаемости увеличивается в два раза и сдвигается с ростом частоты в сторону высоких температур. Температура максимума диэлектрических потерь возрастает с ростом частоты и описывается нелинейной функцией от логарифма частоты. Частотная зависимость комплексной диэлектрической проницаемости не соответствует моделям Дебая и Коула-Коула, которые описывают диэлектрические свойства однородных систем с экспоненциальной зависимостью релаксации параметра поляризации. Частота диэлектрической релаксации, соответствующая максимуму $\operatorname{Im}(\varepsilon)$, имеет активационный характер и хорошо описывается функцией $\ln \omega=A \exp (-\Delta E / k T)$ (рис. 2,b), где энергия активации $\Delta E=0.051 \mathrm{eV}$ и экспонента удовлетворяет условию $\exp (-\Delta E / k T) \ll 1$ при $T<350 \mathrm{~K}$. Ниже этой температуры частоту и время релаксации $(\tau)$ представим в виде

$$
\begin{gathered}
\omega=B \exp \left(\exp \left(-\frac{\Delta E}{k_{\mathrm{B}} T}\right)\right)=B\left(1+\exp \left(-\frac{\Delta E}{k_{B} T}\right)\right) ; \\
\tau=\frac{\tau_{0}}{1+\exp \left(-\frac{\Delta E}{k_{\mathrm{B}} T}\right)} .
\end{gathered}
$$

Энергия активации в (1) соответствует энергии полярона $\left(\varepsilon_{p}\right)$ относительно химического потенциала $(\mu)$, $-\Delta E=\varepsilon_{p}-\mu<0$. Время релаксации пропорционально плотности поляронов $\tau \propto N_{p}$. Если энергия полярона превышает химический потенциал $\varepsilon_{p}>\mu$, то это полярон дырочного типа $N_{\mathrm{ph}}=A /\left(1+\exp \left(\Delta E / k_{\mathrm{B}} T\right)\right)$. Мнимая компонента диэлектрической проницаемости, обусловленная рассеянием поляронов на оптических фононах, пропорциональна $\operatorname{Im}(\varepsilon)_{\max } \propto\left(N_{\mathrm{pe}}+N_{\mathrm{ph}}\right)$. Максимумы диэлектрических потерь увеличиваются с ростом частоты и температуры без магнитного поля и плавно уменьшаются в магнитном поле (рис. $2, b)$ в результате перестройки электронной структуры в магнитном поле. Так, в нулевом поле преобладает спектральный вес поляронов с дырочным типом проводимости с соотношением $N_{\mathrm{ph}} / N_{\mathrm{pe}}=5.5$, а в магнитном поле с электронным типом $N_{\text {pe }} / N_{\text {ph }}=2.3$, что качественно описывает экспериментальные результаты (рис. $2, b$ ).

Температура, при которой диэлектрическая проницаемость резко возрастает и наблюдается максимум диэлектрических потерь, сдвигается в область высоких температур в магнитном поле. Энергия активации возрастает на 5\% в магнитном поле $8 \mathrm{kOe}$. Магнитоемкость
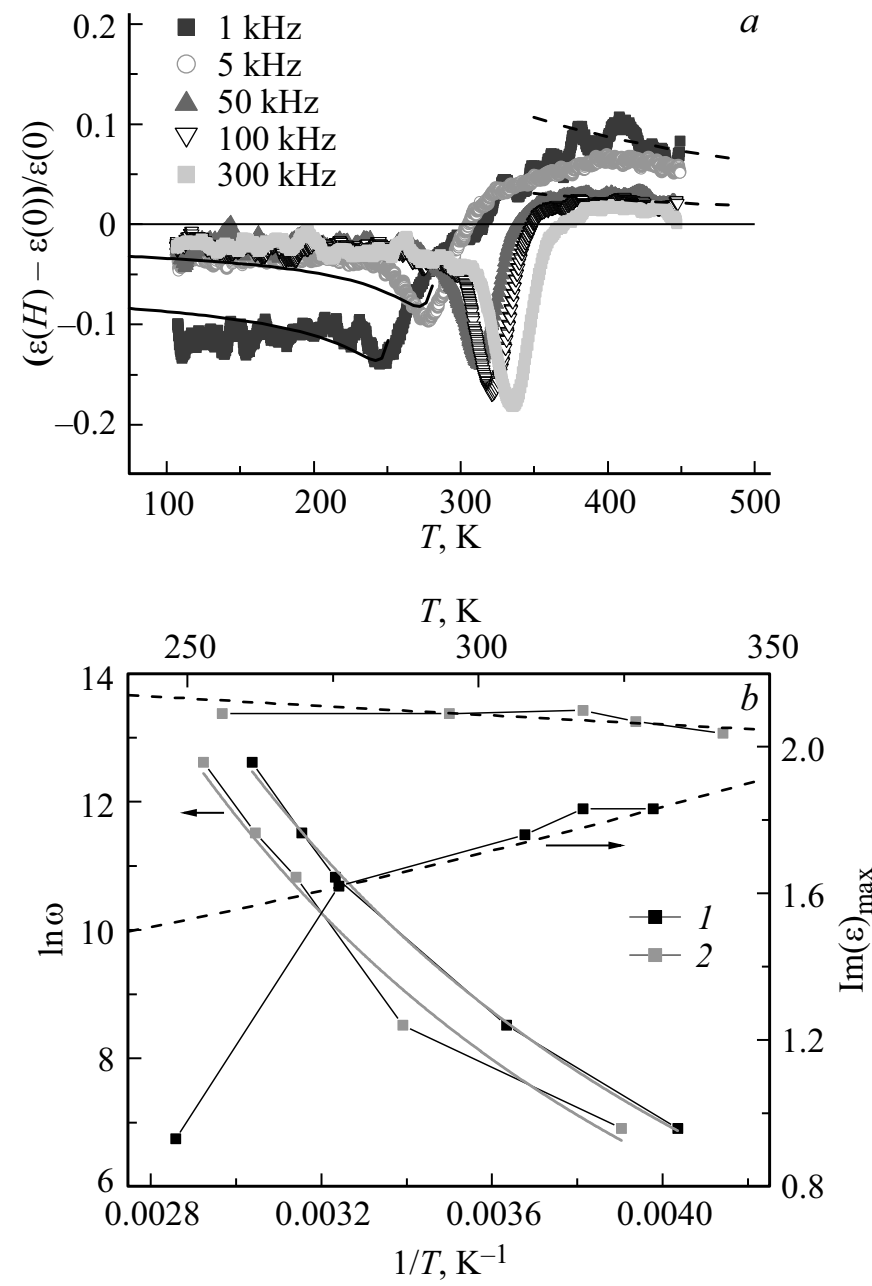

Рис. 2. $a$ ) Магнитоемкость $\delta H$ на частотах $\omega=1,5,50,100$, $300 \mathrm{kHz}$ в магнитном поле $H=8 \mathrm{kOe}$ для состава с $x=0.05$ от температуры. Теоретическое значение магнитоемкости в модели с орбитальным упорядочением (3) (сплошная линия) и в модели Максвелла-Вагнера (4) с $\delta \varepsilon \sim 1 / T^{3 / 2}$ (пунктирная линия). $b$ ) Логарифм частоты релаксации от обратной температуры без поля (1) и в магнитном поле $H=8 \mathrm{kOe}(2)$, подгоночная функция (1) (левая ось). Максимум $\operatorname{Im}(\varepsilon)$ от температуры. Подгоночные функции $\operatorname{Im}(\varepsilon)_{\max }=A N_{\mathrm{pe}}+B N_{\mathrm{ph}}$ (пунктирная линия) (правая ось).

$\delta \varepsilon_{H}=(\operatorname{Re}(\varepsilon(H, T))-\operatorname{Re}(\varepsilon(0, T))) / \operatorname{Re}(\varepsilon(0, T))$ для состава с $x=0.05$ приведена на рис. 2, $a$. При нагревании магнитоемкость меняет знак и уменьшается с ростом частоты.

С ростом концентрации ионов гадолиния возрастает дисперсия неоднородных электронных состояний и локальных магнитных полей. Температурный интервал диэлектрических потерь увеличивается для состава с $x=0.2$ (рис. 3), температуры максимумов мнимой части диэлектрической проницаемости практически не смещаются в магнитном поле, а величина диэлектрических потерь возрастает (рис. 3). В магнитном поле диэлектрическая проницаемость возрастает более резко (рис. 3), в результате магнитоемкость меняет знак с 

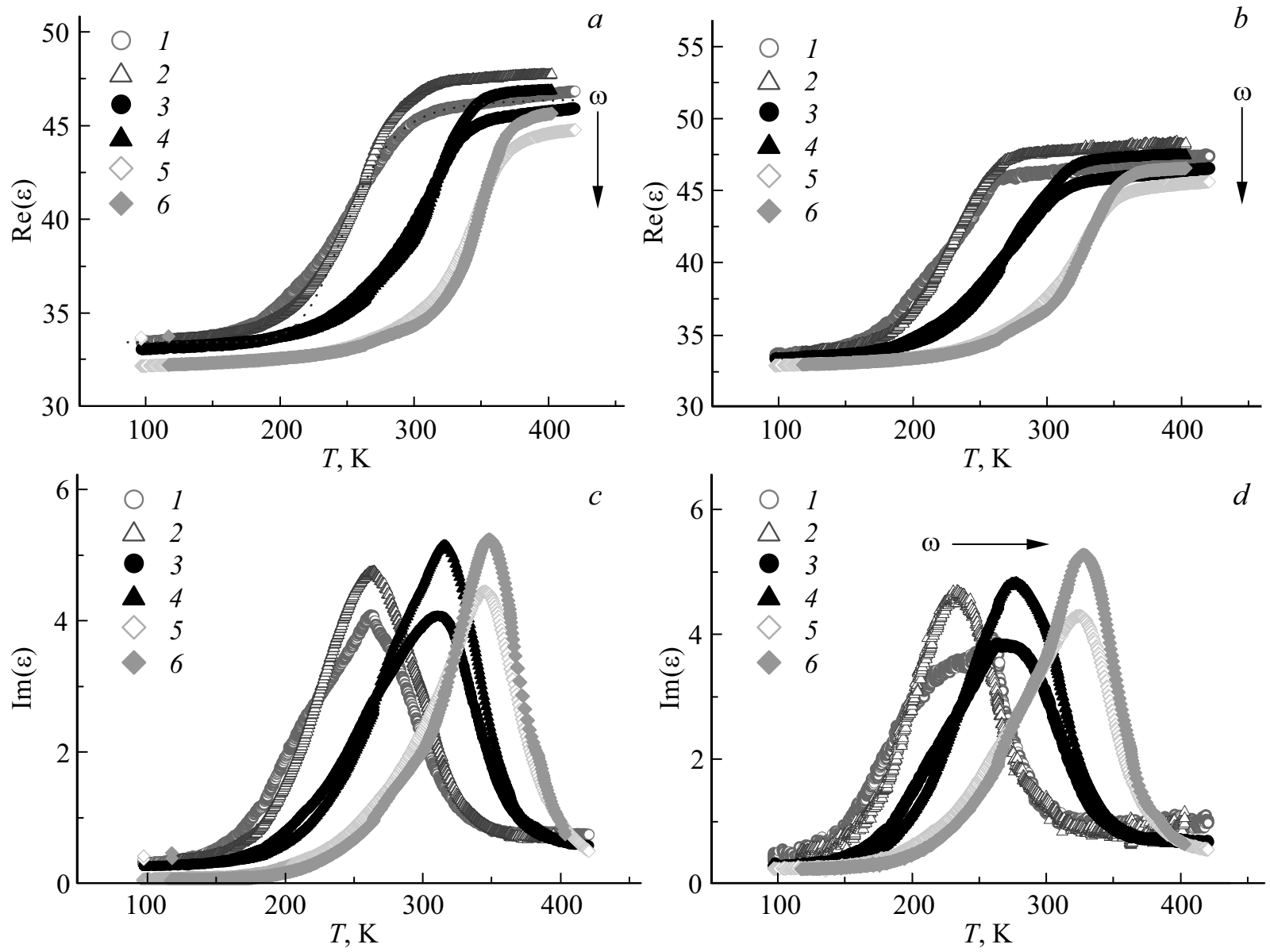

Рис. 3. Действительная $\operatorname{Re}(\varepsilon)(a, b)$ и мнимая $\operatorname{Im}(\varepsilon)(c, d)$ части диэлектрической проницаемости образца $\mathrm{Gd}_{0.2} \mathrm{Mn}_{0.8} \mathrm{Se}_{\text {без }}$ поля $(1,3,5)$ и в магнитном поле $H=8 \mathrm{kOe}(2,4,6)$ на частотах $\omega=5,50,300 \mathrm{kHz}(a, c), \omega=1,10,100 \mathrm{kHz}(b, d)$ от температуры.

отрицательного значения (5-7)\% на положительный $(4-5) \%$ в интервале температур (140-400) K (рис. 4, $a$ ).

Температуры максимумов диэлектрических потерь возрастают в пределах $1 \%$ в магнитном поле и растут с увеличением частоты. Частота релаксации описывается экспоненциальной зависимостью (1) с энергией активации $\Delta E=0.035 \mathrm{eV}$. Диэлектрические потери связаны с рассеянием поляронов дырочного типа и функция $\operatorname{Im}(\varepsilon)_{\max }=A N_{\text {ph }}$ удовлетворительно описывает экспериментальные данные (рис. $4, b$ ).

Изменение диэлектрической проницаемости по температуре сопровождается отклонением магнитной восприимчивости от закона Кюри-Вейсса. На рис. 5 представлена обратная величина магнитной восприимчивости от температуры. Выше температуры $T=250 \mathrm{~K}$ обратная магнитная восприимчивость хорошо описывается законом Кюри-Вейсса с парамагнитной температурой $\Theta$, имеющей отрицательное значение и возрастающей от $\Theta_{P}=-350 \mathrm{~K}$ для $\mathrm{MnSe}$ до $\Theta_{P}=-98 \mathrm{~K}$ для $x=0.2$, что свидетельствует об образовании ферромагнитного обмена при замещении марганца гадолинием. Это уменьшение вызвано локализацией электронов в кластерах с ионами гадолиния и образованием ферромагнитного обменного взаимодействия на интерфейсе $\mathrm{Mn}-\mathrm{Gd}$ в результате кинетического обменного взаимодействия.

Эффективный магнитный момент увеличивается с ростом концентрации от $5.9 \mu_{\mathrm{B}}$ для $\mathrm{MnSe}$ до $6.39 \mu_{\mathrm{B}}$ в $\mathrm{Mn}_{0.8} \mathrm{Gd}_{0.2} \mathrm{Se}$ (рис. 6,a). Причем величина магнитного момента на $(1-3) \%$ больше, по сравнению с линейным ростом $\mu^{\mathrm{ef}}(x)=(1-x) \mu_{\mathrm{Mn}}^{\mathrm{ef}}+x \mu_{\mathrm{Gd}}^{\mathrm{ef}}$ суммы эффективных моментов ионов марганца и гадолиния. Снятие вырождения $\operatorname{tg}_{2 g}$ электронных состояний приводит к понижению локальной симметрии кластеров ионов марганца, к изменению $g$-фактора и эффективного магнитного момента $\mu^{\mathrm{ef}}=g \sqrt{(S(S+1)} \mu_{\mathrm{B}}$. Концентрация таких кластеров, содержащих ион гадолиния в окружении ионов марганца с магнитными моментами $\mu_{\mathrm{Mn}}^{\mathrm{ef} *}$, равна $c_{g}=z x(1-x)^{z-1}$, и изменение эффективного момента от концентрации сводится к виду $\mu^{\mathrm{ef}}(x) / \mu_{\mathrm{Mn}}^{\mathrm{ef}}=1-x-(1-\lambda) c_{g}+\gamma x$, где $\lambda=\mu_{\mathrm{Mn}}^{\mathrm{ef} *} / \mu_{\mathrm{Mn}}^{\mathrm{ef}}$, $\gamma=\mu_{\mathrm{Gd}}^{\mathrm{ef}} / \mu_{\mathrm{Mn}}^{\mathrm{ef}}=1.34$. Подгоночная функция описывает экспериментальные результаты с параметром $\lambda=1.055$, что соответствует увеличению $g$-фактора до 2.11 . В неупорядоченной системе индуцируется орбитальный магнитный момент, который взаимодействует со спиновым в результате спин-орбитального взаимодействия. 

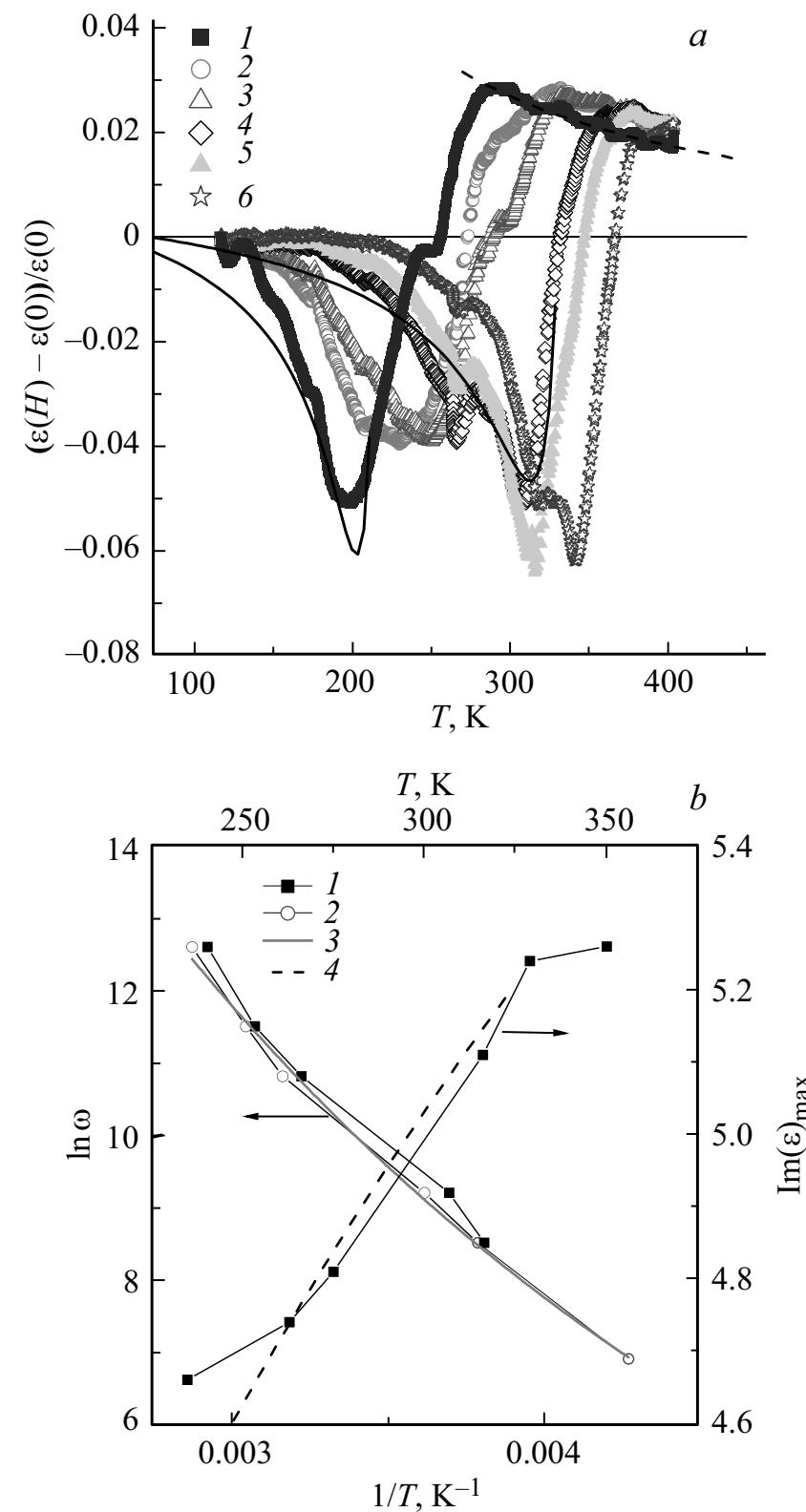

Рис. 4. $a)$ Магнитоемкость $\delta \varepsilon_{H}$ на частотах $\omega=1(1), 5$ (2), 10 (3), 50 (4), 100 (5), 300 (6) kHz в магнитном поле $H=8 \mathrm{kOe}$ для состава с $x=0.2$ от температуры. Теоретическое значение магнитоемкости в $\mathrm{Gd}_{0.2} \mathrm{Mn}_{0.8} \mathrm{Se}$ в модели с орбитальным упорядочением (3) (сплошная линия) и в модели Максвелла-Вагнера (4) с $\delta \varepsilon \sim 1 / T^{3 / 2}$ (пунктирная линия). $b)$ Логарифм частоты релаксации от обратной температуры без поля (1) и в магнитном поле $H=8 \mathrm{kOe}(2)$, подгоночная функция (1) (кривая 3) (левая ось). Максимум $\operatorname{Im}(\varepsilon)_{\max }$ от температуры и подгоночная функция $\operatorname{Im}(\varepsilon)_{\max }=A N_{\mathrm{ph}}(4)$ (правая ось) (b).

\section{3. Модель}

Трехвалентный селенид гадолиния согласно теоретическим расчетам LSDA + U [18] является металлом, зона проводимости которого обусловлена $5 d$-электронами и верхняя хабардовская подзона $4 f$-электронов нахо- дится на $2 \mathrm{eV}$ выше уровня Ферми. Ширина зоны проводимости составляет $8 \mathrm{eV}$. Замещение двухвалентного марганца трехвалентными ионами гадолиния приводит к электронному допированию и к вырождению $t_{2 g}$ электронных состояний, которое снимается ян-теллеровским и спин-орбитальным взаимодействием. Тип носителей тока в $\mathrm{MnSe}$ - дырки, которые взаимодействуют с оптическими модами колебаний и образуют решеточные поляроны, которые закрепляются на интерфейсе $\mathrm{Mn}-\mathrm{Gd}$. Пиннингование поляронов понижает локальную симметрию и индуцирует орбитальный магнитный момент на узле. Для манганитов найдено уменьшение диэлектрической проницаемости в результате конденсации одной из компонент трехкратно вырожденной $T_{1 \mathrm{~g}}$ моды колебаний в центре или на границах зоны Бриллюэна [19]. Этот механизм приводит к изменению диэлектрической проницаемости в твердом растворе $\mathrm{Mn}_{1-x} \mathrm{Gd}_{x} \mathrm{Se}$.

Константу спин-орбитального взаимодействия $(\alpha)$ для иона в октаэдрическом приближении оценим из соотношения $\alpha=2(1-g / \Delta)$, где $\Delta-$ величина расщепления $t_{2 g}$-состояний, которая для $3 d$-металлов со структурой

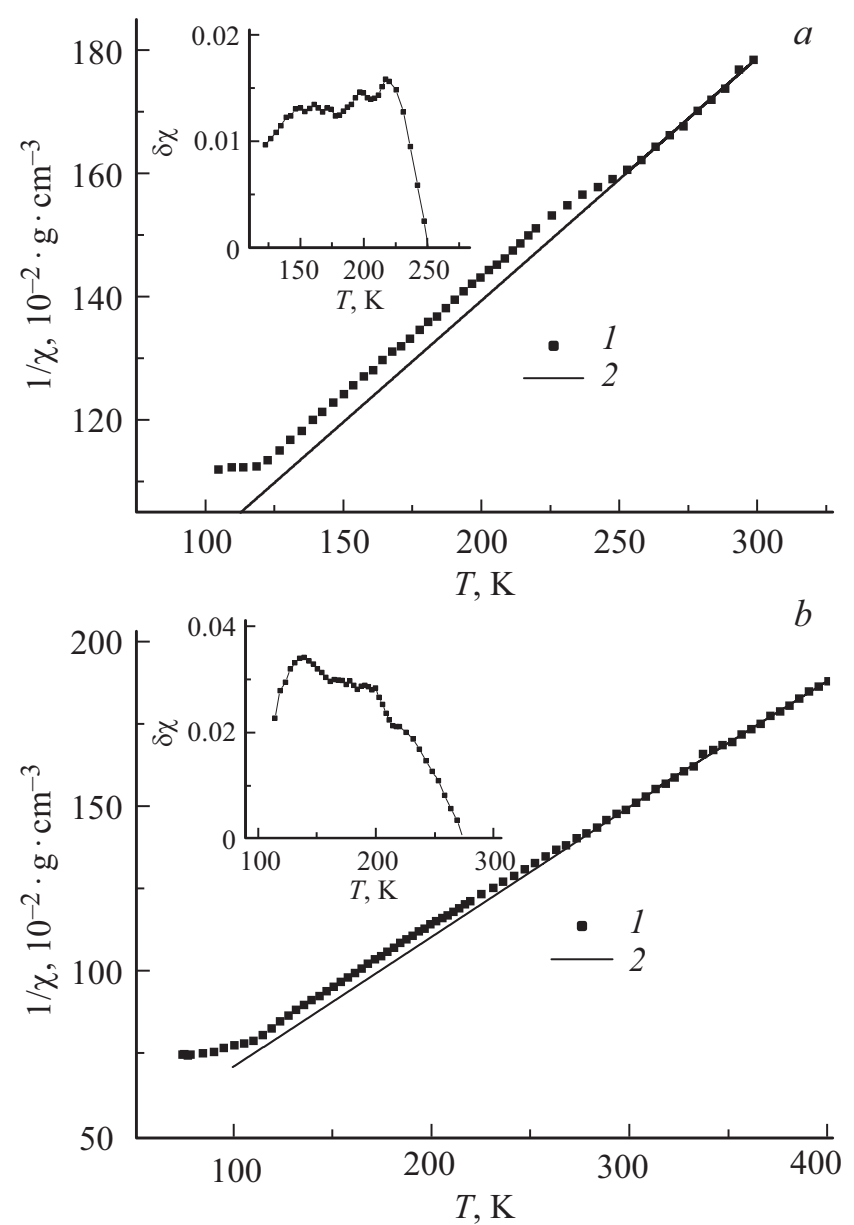

Рис. 5. Обратная восприимчивость от температуры для двух составов $x=0.05(a)$ и $0.2(b)$. На вставках — разница магнитных восприимчивостей Кюри-Вейсса (2) и экспериментальных значений $(1) \delta \varepsilon=\left(\chi^{C W}-\chi^{E X}\right) / \chi^{E X}$ от температуры. 


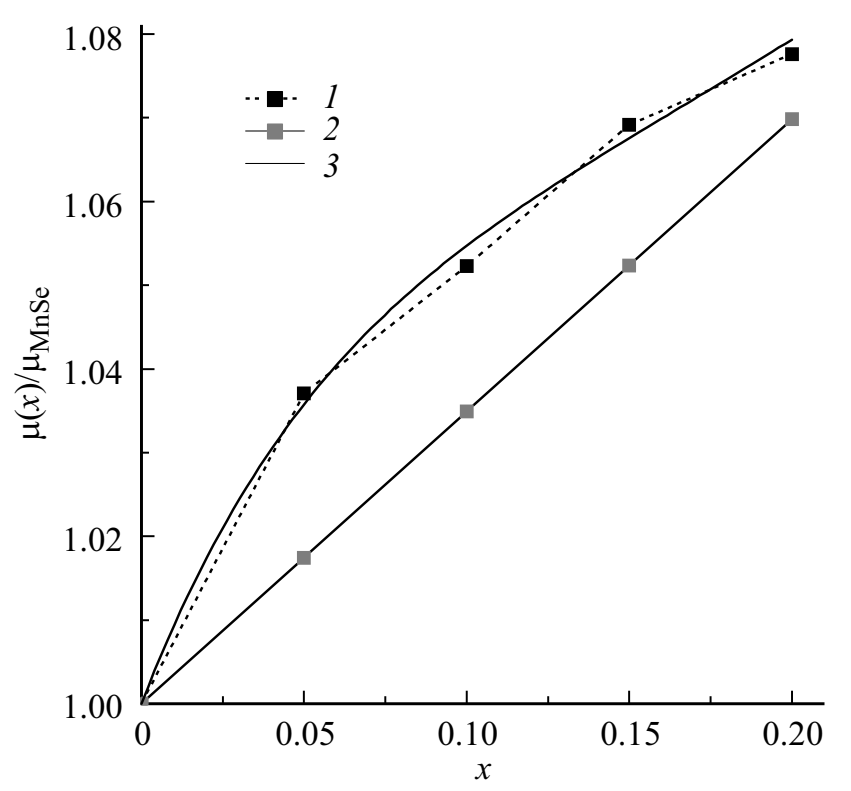

Рис. 6. Эффективный магнитный момент катионов $\mu^{\mathrm{ef}}(x) / \mu_{\mathrm{Mn}}^{\mathrm{ef}}$ в твердом растворе $\mathrm{Gd}_{X} \mathrm{Mn}_{1-X} \mathrm{Se}$ от концентрации ионов гадолиния: эксперимент (1), сумма эффективных моментов $\mu^{\mathrm{ef}}(x) / \mu_{\mathrm{Mn}}^{\mathrm{ef}}=1-x+x \mu_{\mathrm{Gd}}^{\mathrm{ef}} / \mu_{\mathrm{Mn}}^{\mathrm{ef}}$ (2) и модельных вычислений $\mu^{\mathrm{ef}}(x) / \mu_{\mathrm{Mn}}^{\mathrm{ef}}=1-x-(1-\lambda) c_{g}+1.34 x$, где $\lambda=\mu_{\mathrm{Mn}}^{\mathrm{ef}} * \mu_{\mathrm{Mn}}^{\mathrm{ef}}(3)$.

перовскита варьируется в пределах $\Delta=0.1-0.15 \mathrm{eV}$ и $\alpha \sim 10 \mathrm{meV}$. Константа одноосной магнитной анизотропии $D=\alpha^{2} / \Delta \sim 10^{-3} \mathrm{eV}$ и поле анизотропии на порядок меньше обменного поля $H_{A} / H_{\mathrm{ex}}=D / z J \sim 0.1$. Появление продольной компоненты углового магнитного момента сопровождается магнитной и диэлектрической анизотропией в плоскости, это следует из анизотропного распределения электронной плотности волновых функций электрона. Используем простое соотношение между компонентами диэлектрической проницаемости $\varepsilon_{x x}>\varepsilon_{y y}$ для проекций орбитального магнитного момента $L^{z}=+1$ и $\varepsilon_{x x}<\varepsilon_{y y}$ для $L_{z}=-1$ и $L_{z}=0, \varepsilon_{x}=\varepsilon_{y y}$.

Для малых концентраций $x<x_{c} \quad\left(x_{c}=0.17-\right.$ концентрация протекания) основной вклад в магнитные и диэлектрические свойства дадут кластеры ионов гадолиния, в ближайшем окружении которого находятся только ионы марганца. Ниже $250 \mathrm{~K}$ в окрестности этих кластеров возникают локальная деформация решетки и замораживание орбитальных магнитных моментов со случайной анизотропией диэлектрической проницаемости. Нанообласти имеют ближний ферромагнитный порядок и изменение ориентации дипольных моментов кластеров $\left(M_{L}\right)$ в магнитном поле $H$ опишем в модели суперпарамагнетика.

Представим энергию суперпарамагнитных частиц в виде $W=M_{L} H \cos \theta+M_{L} H_{A} \cos (\gamma-\theta)$, где угол $\theta$ задает направление магнитного момента относительно магнитного поля, а $\gamma-$ угол между магнитным полем и полем анизотропии, $H_{A}-$ поле анизотропии. Равновесное направление орбитального момента определяется выражением $\operatorname{tg} \theta=H_{A} \sin \gamma /\left(H+H_{A} \cos \gamma\right)$. Изменение орбитального момента всех частиц в магнитном поле представим в виде

$$
\begin{aligned}
M_{L}^{0} \Delta \theta & =M_{L}^{0}\left(\langle\cos \theta\rangle_{H}-\langle\cos \theta\rangle_{0}\right) \\
& =M_{L}^{0}\left(\sum_{i} \frac{1}{\sqrt{1+\frac{H_{A}^{2} \sin ^{2} \gamma_{i}}{\left(H+H_{A} \cos \gamma_{i}\right)}}}-\frac{1}{1+\operatorname{tg}^{2} \gamma_{i}}\right) .
\end{aligned}
$$

Относительное изменение диэлектрической проницаемости в магнитном поле пропорционально анизотропии диэлектрической проницаемости $\Delta \varepsilon=\varepsilon_{x x}-\varepsilon_{y y}$ и изменению орбитального момента в магнитном поле :

$$
\begin{aligned}
& \delta \varepsilon=\Delta \varepsilon\left(M_{L}(H)-M_{L}(0)\right)=\Delta \varepsilon m_{L}\left(1-T / T_{c}\right)^{\beta} \\
& \times\left(\sum_{i} \frac{1}{\sqrt{1+\sin ^{2} \gamma_{i}\left[\frac{\lambda}{\left(1-T / T_{c, B}\right)^{2}}+\cos \gamma_{i}\right]^{2}}}-\frac{1}{\sqrt{1+\operatorname{tg}^{2} \gamma_{i}}}\right),
\end{aligned}
$$

где $\lambda=H / H_{A}$. В приближении среднего поля намагниченность в области перехода имеет вид $M=m_{L}\left(1-T / T_{C}\right)^{\beta}$, где $\beta=0.5$. Поле анизотропии также имеет степенную зависимость: $H_{A}=D\left(1-T / T_{c, B}\right)^{n}$. Для $x=0.05$ на частоте $\omega=1 \mathrm{kHz}$ экспериментальные данные по магнитоемкости (рис. 2) удовлетворительно описываются функцией (3) со следующими параметрами: орбитальный момент кластера $m_{L}=0.1$, $\lambda=0.01, n=1.5$, температура пиннингования поляронов с образованием орбитального магнитного момента $T_{c, B}=252 \mathrm{~K}$. С ростом частоты температура закрепления решеточных поляронов на интерфейсе $\mathrm{Mn}-\mathrm{Gd}$ возрастает и минимумы на зависимости $\delta \varepsilon(T)$ качественно описываются функцией (3). При переходе к зонному типу проводимости подвижность носителей тока возрастает и магнитоемкость обусловлена механизмом Максвелла-Вагнера, когда неоднородность смешивает реальные и мнимые моды диэлектрического отклика, продольные и поперечные моды колебаний. Неоднородность также вызывает магнитосопротивление на постоянном токе в результате смешивания холловского сопротивления с продольным сопротивлением, обусловливая линейную зависимость от поля. В 2Dмодели двухкомпонентной сильно неоднородной среды с проводимостью $\sigma_{1}=0, \varepsilon_{1}=\varepsilon$ и $\sigma_{2}=\sigma, \varepsilon_{2}=0$ для случая равных концентраций получено точное выражение для продольной диэлектрической проницаемости [13]:

$$
\varepsilon_{x x}(\omega)=\varepsilon \frac{(1+i \omega \tau)}{\sqrt{i \omega \tau} \sqrt{(1+i \omega \tau)^{2}-(\omega \tau \beta)^{2}}},
$$

где $\beta=\mu H$, где $\mu-$ подвижность носителей заряда в магнитном поле. Реальная и мнимая компоненты диэлектрической проницаемости растут в магнитном поле и при $\omega \tau \beta<1, \omega \tau \approx 1$ зависимость диэлектрической 
проницаемости от подвижности и магнитного поля хорошо описывается степенной функцией $\varepsilon_{x x}(\beta) / \varepsilon=A \beta^{0.7}$. Из магнитоемкости оценим подвижность носителей в данной модели $\delta \varepsilon=\left(\varepsilon_{x x}(\beta)-\varepsilon_{x x}(0)\right) / \varepsilon(0)=0.6(\mu H)^{0.7}$, величина которой составляет $\mu \approx 0.01 T^{-1}$ на частоте $\omega=1 \mathrm{kHz}$. При температуре выше $250 \mathrm{~K}$ сохраняется ближний орбитальный порядок и магнитоемкость обусловлена двумя конкурирующими механизмами: уменьшением диэлектрической проницаемости в магнитном поле в результате усиления корреляций орбитальных магнитных моментов электронов и ростом $\varepsilon$ за счет эффекта Максвелла-Вагнера. Подвижность носителей заряда уменышается при высоких температурах $T>350 \mathrm{~K}$ за счет рассеяния на фононах согласно степенной функции $\mu=A / T^{3 / 2}$. Магнитоемкость, обусловленная изменением подвижности, удовлетворительно описывает экспериментальные результаты (рис. 2).

Для концентраций, превышающих концентрацию протекания $x>x_{c}$, на интерфейсе $\mathrm{Mn}-\mathrm{Gd}$ образуется дальний орбитальный порядок, результирующий орбитальный магнитный момент которого отличен от нуля. Основной вклад в магнитоемкость обусловлен анизотропией диэлектрической проницаемости в окрестности интерфейса $\mathrm{Gd}-\mathrm{Mn}$, которая регулируется магнитным полем за счет спин-орбитальной связи. Экспериментальные данные удовлетворительно описываются функцией (3) с параметрами: $\beta=0.33, n=2, M_{L}(0)=0.025$. При более высоких температурах $T>250 \mathrm{~K}$ магнитоемкость вызвана уменьшением подвижности носителей тока $\delta \varepsilon(T)=A / T^{3 / 2}$ в результате рассеяния их на фононах.

\section{4. Заключение}

В твердых растворах $\mathrm{Gd}_{x} \mathrm{Mn}_{1-x} \mathrm{~S}$ обнаружен магнитоемкостный эффект при температурах, в несколько раз превышающих температуру Нееля, со сменой знака по температуре. Логарифм частоты релаксации диэлектрических потерь экспоненциально растет при нагревании и зависит от магнитного поля. Найден нелинейный рост эффективного магнитного момента от концентрации, обусловленный вкладом орбитального магнитного момента ионов марганца вблизи интерфейса $\mathrm{Mn}-\mathrm{Gd}$. Установлена корреляция температур, при которых магнитная восприимчивость увеличивается, а диэлектрическая проницаемость уменьшается.

Экспериментальные данные объясняются в модели с орбитальным упорядочением. При температуре ниже температуры Дебая поляроны пиннингуются на интерфейсе с появлением орбитального магнитного момента на узле и анизотропии диэлектрической проницаемости. Для концентраций ионов гадолиния, меньших концентрации протекания, образуется орбитальное „стекло“, а для более высоких концентраций индуцируется дальний орбитальный порядок на интерфейсе. Изменение орбитальных корреляций магнитного углового момента в магнитном поле меняет анизотропию диэлек- трической проницаемости. Делокализация электронов и переход к зонному типу проводимости приводит к положительной магнитоемкости в результате эффекта Максвелла-Вагнера.

\section{Список литературы}

[1] W. Eerenstein, N.D. Mathur, J.F. Scott. Nature 442, 759 (2006).

[2] R.J. Zeches, M.D. Rossell, J.X. Zhang, A.J. Hatt, Q. He, C.H. Yang. Science 326, 977 (2009).

[3] A. Nicola Spaldin, Sang-Wook Cheong, Ramamoorthy Ramesh. Physics Today 63, 38 (2010).

[4] А.П. Пятаков, А.К. Звездин. УФН 182, 583 (2012).

[5] J.C. Yang, Q. He, S.J. Suresha, C.Y. Kuo, C.Y. Peng, R.C. Haislmaier, M.A. Motyka. Phys. Rev. Lett. 109, 247606 (2012).

[6] А.К. Звездин, А.П. Пятаков. УФН, 179, 897 (2009).

[7] T. Kambe, Y. Fukada, J. Kano, T. Nagata, H. Okazaki, T. Yokoya, S. Wakimoto, K. Kakurai, N. Ikeda. Phys. Rev. Lett. 110, 117602 (2013).

[8] A. Scaramucci, E. Bousquet, M. Fechner, M. Mostovoy, N.A. Spaldin. Phys. Rev. Lett. 109, 197203 ( 2012).

[9] М.Ю. Каган, К.И. Кугель. УФН 171, 577 (2001).

[10] K.I. Kugel, A.L. Rakhmanov, A.O. Sboychakov, D.I. Khomskii. Phys. Rev. B 78, 155113 (2008).

[11] J.C. Maxwell. Treatise on Electricity and Magnetism, 3rd ed. Dover, N. Y. (1991)

[12] G. Catalan. Appl. Phys. Lett. 88, 102902 (2006).

[13] M.M. Parish, P.B. Littlewood. Phys. Rev. Lett. 101, 166602 (2008).

[14] M. Prasad, A.K. Pandit, T.H. Ansari, R.A. Singh. Mater. Chem. Phys. 30, 13 (1991).

[15] M.M.R. Costa, M.J.M de Almeida, W.J. Nuttall, W.G. Stirling, C.C. Tang, J.B. Forsyth, M.J. Cooper. J. Phys.: Condens. Matter 8, 2425 (1996).

[16] С.С. Аплеснин, О.Б. Романова, М.В. Горев, А.Д. Васильев, О.Ф. Демиденко, Г.И. Маковецкий, К.И. Янушкевич. ФТТ 7, 1296 (2012).

[17] С.С. Аплеснин, А.М. Живулько, К.И. Янушкевич. В кн.: Перспективные материалы и технологии. Витебск (2017). C. 256-279.

[18] Dipta Bhanu Ghosh, Molly De, S.K. De. J. Phys.: Condens. Matter. 15, 7095 (2003).

[19] I. Fedorov, J. Lorenzana, P. Dore, G. De Marzi, P. Maselli, P. Calvani, S.-W. Cheong, S. Koval, R. Migoni. Phys. Rev. B 60, 11875 (1999). 